\title{
Consumo de Drogas y Violencia en el Noviazgo en Estudiantes Universitarios del Sur de Chile
}

\author{
Drug abuse and dating violence in university students in southern Chile
}

\author{
Claudia Saldivia \\ Universidad Santo Tomás, Chile. \\ $\&$ \\ Beatriz Vizcarra \\ Universidad de La Frontera, Chile.
}

(Rec: 26 de mayo de 2011 / Acep: 25 de abril de 2012)

\begin{abstract}
Resumen
La presente investigación tuvo por objetivo describir la relación entre consumo de drogas y violencia en el noviazgo, en jóvenes universitarios del sur de Chile. La muestra estuvo compuesta por 205 estudiantes, quienes respondieron a dos instrumentos: un cuestionario de detección de consumo problemático de alcohol y otras drogas y un cuestionario de violencia de pareja en jóvenes. Los resultados obtenidos muestran una relación significativa y directa entre consumo de drogas y violencia en el noviazgo. Específicamente se obtuvo una asociación significativa entre consumo y ser víctima de violencia psicológica y física. Se discuten estos resultados en sus implicancias teóricas y prácticas, señalando las limitaciones de la investigación y sugerencias para futuros estudios.
\end{abstract}

Palabras clave: Drogas, violencia en el noviazgo, estudiantes universitarios.

\begin{abstract}
The present investigation aimed to describe the relationship between drug use and dating violence in university students of southern Chile. A sample of 205 Students completed two instruments: a questionnaire for detecting problematic use of alcohol and other drugs and a questionnaire for detecting intimate partner violence in young people. Results show a significant and direct relationship between drugs and alcohol use and dating violence. Regarding consumption and types of violence, a significant association between consumption and being victim of psychological and physical violence was found. Results are discussed as to their theoretical and practical implications, indicating the limitations of this research and suggestions for future studies. Keywords: Drug use, dating violence, university students.
\end{abstract}

\section{Introducción}

En el estudio del comportamiento y hábitos de vida de los jóvenes chilenos, surgen como problemáticas importantes el consumo de alcohol y otras drogas, la dificultad de acceso al trabajo, y la violencia, entre otros (Sexta Encuesta Nacional de la Juventud, Instituto Nacional de la Juventud, INJUV, 2009; Noveno Estudio Nacional de Drogas, Servicio Nacional para la Prevención y Rehabilitación del Consumo de Drogas y Alcohol, SENDA, 2011). Este último estudio revela que en el tramo de 19 a 25 años, un $1.3 \%$ de los jóvenes consume cocaína, un $12.3 \%$ consume marihuana, un $27.4 \%$ tabaco y un $55.4 \%$ alcohol.

El consumo de alcohol y otras drogas tiene importantes efectos en el desarrollo de vida de los jóvenes, ya que produce consecuencias a nivel individual, detrimento de la salud física y psicológica; deterioro de las funciones cognitivas y alteraciones emocionales (Espada, Méndez, Griffin \& Botvin, 2003). Las consecuencias a nivel familiar y psicosocial son: aislamiento familiar, social, alteración de la dinámica familiar, conductas de riesgo como conductas delictivas y promiscuas, involucramiento en microtráfico

Correspondencia: Claudia Saldivia, Escuela de Psicología, Universidad Santo Tomás, Rodríguez 060, Temuco, Chile. Correo electrónico: claudiasaldivia@ santotomas.cl; csaldiviam@gmail.com.

La presente investigación fue financiada parcialmente con fondos de la Dirección de Investigación de la Universidad de La Frontera, Proyecto DIUFRO D110-2009 
y tráfico de sustancias, aumento de la violencia interpersonal e intentos de suicidio. También se describen consecuencias económicas para el consumidor, su familia y la sociedad (Rees \& Valenzuela, 2003; Rodríguez, Fernández, Hernández \& Ramírez, 2006).

En cuanto a la violencia en el noviazgo, de acuerdo a la Sexta Encuesta Nacional de la Juventud realizada por el INJUV, Chile, en el año 2009, un 16.9\% de los jóvenes encuestados señala la existencia de violencia psicológica en su pareja actual, un 7.7\% reporta violencia física y un $0.8 \%$ indica la existencia de violencia sexual.

El impacto que causa la violencia de pareja en los jóvenes se encuentra relacionado con una disminución de la calidad de vida y bienestar psicosocial; algunas de las consecuencias descritas son bajo rendimiento académico, deserción escolar, embarazo precoz, transmisión de enfermedades sexuales y trastornos alimentarios (Foshee, Bauman, Linder, Rice \& Wilcher, 2007; González, Muñoz \& Graña, 2003). Específicamente, en las víctimas se observan trastornos depresivos, baja autoestima, inseguridad y aislamiento, y el riesgo de ser victimizadas en sus relaciones de pareja en la adultez. En los perpetradores, la literatura internacional reporta rechazo social y el riesgo de repetir estas conductas en la vida adulta. (Hernando, 2007; Rivera-Rivera, Allen, Rodríguez-Ortega, Chávez-Ayala \& Lazcano-Ponce, 2006; Servicio Nacional de la Mujer, Gobierno de Chile, 2004; Vézina \& Hébert, 2007).

En cuanto a la dinámica de la violencia, en general las investigaciones muestran que la violencia en el noviazgo, a diferencia de la violencia en parejas adultas, se caracterizaría por ser bidireccional o cruzada, ya que en estas relaciones se daría un mayor equilibrio de poder, al no existir dependencia económica ni cohabitación (Aguirre \& García, 1997; González et al., 2003; Poo \& Vizcarra, 2008).

Investigaciones internacionales señalan que ambas temáticas, consumo de drogas y violencia en el noviazgo, se encuentran relacionadas (Guzmán, Esparza, Alcántara, Escobedo \& Henggeler, 2009; Muñoz-Rivas, GámezGuadix, Graña \& Fernández, 2010; Wolfe \& Feiring, 2000). González (2008), en un meta-análisis de investigaciones internacionales sobre el tema, confirma la existencia de una relación significativa entre consumo de alcohol y violencia en el noviazgo, ya sea que el consumo esté dado como factor de riesgo, o como consecuencia de la violencia en la pareja. Si bien esta misma autora menciona que algunos estudios no encuentran una relación significativa entre consumo de alcohol y violencia en el noviazgo, estas investigaciones presentarían deficiencias metodológicas en cuanto a la medición del consumo de alcohol.

El consumo de drogas ha sido categorizado como un factor de riesgo en la victimización, ya que altera el juicio de realidad e impide reconocer y evitar situaciones de peligro, especialmente en casos de agresiones sexuales (Foshee et al, 2007; Hernando, 2007; Vézina \& Hébert, 2007).También ha sido descrito como factor de riesgo, tanto en la victimización como la perpetración de la violencia psicológica, pero solo si el consumo es moderado o severo; ya que alteraría la percepción de los patrones de interacción y comunicacionales en la pareja (Guzmán et al., 2009).

En el caso de los agresores, el consumo habitual también altera el juicio de realidad, disminuyendo la inhibición de impulsos, lo que llevaría a mayor riesgo de cometer agresiones, especialmente físicas y sexuales (González, 2008, Muñoz-Rivas et al., 2010).

Por otra parte, el consumo de drogas también se ha señalado como una consecuencia de la violencia de pareja en jóvenes, junto con otros síntomas clínicos y psicosociales, como la depresión, baja autoestima, bajo rendimiento académico y deserción escolar (Anglin, Song \& Lunghofer, 1995, en Vizcarra \& Poo, 2007; González et al., 2003).

No obstante, es necesario señalar que la relación entre el consumo de drogas y la violencia en el noviazgo no se expresa en una relación causa - efecto, sino como parte de un complejo fenómeno multicausal, existiendo en la base de ambos fenómenos, factores comunes que influyen en la aparición y mantención de ambas problemáticas. Algunos factores son la violencia intrafamiliar en la infancia, ya sea como testigo de violencia entre los padres o víctima de maltrato infantil, escasa supervisión parental y/o malas relaciones con los padres, contexto comunitario de violencia, entre otros (Vézina \& Hébert, 2007; Wolfe \& Feiring, 2000). Algunos autores señalan que ambos fenómenos covarían y serían parte de un "síndrome de comportamiento problema" (Jessor, 1993), que incluye además otras conductas de riesgo como embarazo no deseado, conductas antisociales y conductas sexuales riesgosas (Jessor, 1993; Muñoz-Rivas et al., 2010).

Las investigaciones en ambas temáticas en universitarios chilenos, muestran que el consumo de drogas ilícitas en este sector es de un $5.68 \%$, específicamente $4.31 \%$ utiliza drogas de uso médico como tranquilizantes y analgésicos, $42.89 \%$ consume tabaco y $59.11 \%$ consume alcohol (Informe sobre uso de drogas en estudiantes de educación superior, SENDA, 2004), siendo este grupo uno de los de más alto riesgo en el consumo de drogas ilícitas y abuso de alcohol (Séptimo Estudio Nacional de Drogas, SENDA, 2006).

Por otra parte un estudio de prevalencia realizado en estudiantes universitarios del sur de Chile, arroja que un $81.6 \%$ de los estudiantes presenta un consumo no problemático de alcohol y drogas; un $16.3 \%$ presentan un consumo moderado y un $2 \%$ un consumo severo. Estos últimos niveles implican riesgos y dependencia y requieren intervención especializada (Vizcarra, 2007).

En cuanto a la violencia en el noviazgo, uno de los primeros estudios en universitarios chilenos, realizado por Aguirre y García (1997), refiere porcentajes de 51\% de violencia psicológica y $24 \%$ de violencia física en las 
relaciones de pareja en universitarios. Estas cifras coinciden con las reportadas posteriormente por Vizcarra \& Poo (2011), de 57\% de violencia psicológica, y $26 \%$ de violencia física y son más elevadas que las reportadas por el Instituto Nacional de la Juventud (2009), lo cual puede relacionarse con la forma de medición, ya que en este último estudio la medición de la violencia física, psicológica y sexual se hizo sólo en base a 5 ítems a diferencia de los estudios anteriores en que utilizaron escalas compuestas de varias preguntas.

Dada la escasa investigación respecto de la relación entre consumo de drogas y violencia en el noviazgo en los jóvenes en Chile, se consideró relevante investigar al respecto, especialmente si se considera la alta prevalencia de ambos fenómenos, que constituyen problemas psicosociales significativos (Hernando, 2007; Muñoz-Rivas et al., 2010; SERNAM, 2004). A partir de lo anterior, el objetivo de la presente investigación es describir la relación entre consumo de drogas y las diversas formas de violencia en el noviazgo en universitarios chilenos.

\section{Método}

\section{Participantes}

En este estudio participaron 205 estudiantes de una población total de 748 estudiantes matriculados de las carreras de pregrado de la Universidad Arturo Prat, Región de la Araucanía, Chile, en el año 2010. Los grupos curso fueron seleccionados a través de un muestreo no probabilístico por conveniencia, dada la facilidad de acceso a los participantes, quienes constituyen una población cautiva. La participación fue anónima y voluntaria.

Los criterios de inclusión de la muestra fueron los siguientes:

- $\quad$ ser estudiante de carreras de pregrado (Ingeniera Civil Informática, Ingeniería Civil Industrial, Derecho, Enfermería, Kinesiología y Psicología) de la Universidad Arturo Prat, Región de la Araucanía, Chile, durante el año 2010, y tener entre 18 a 29 años.

- estar o haber estado en una relación de pareja durante los doce meses previos a la recolección de datos.

En tanto, los criterios de exclusión fueron: no ser estudiante de las carreras antes mencionadas, de la Universidad Arturo Prat, Región de La Araucanía, Chile; tener menos de 18 o más de 29 años de edad; y no estar o haber estado en una relación de pareja durante los doce meses anteriores a la recolección de datos.

\section{Instrumentos}

a) Cuestionario de Detección de Consumo Problemático de Alcohol y Drogas en Adolescentes (DEP-ADO). Creado por Landry, Tremblay, Guyon, Bergeron \& Brunelle
(2004). Este instrumento consta de 17 ítems, los cuales se organizan en 3 factores: Factor 1: Consumo de alcohol y marihuana, compuesto por 7 ítems. Factor 2: Consumo de otras drogas, compuesto por 4 ítems. Factor 3: Consecuencias, compuesto por 6 ítems. Los ítems se presentan en formato Likert con seis valores desde "No ha consumido" (0) hasta "todos los días" (5) y formato dicotómico ("sí" o "no"; 1 y 0 respectivamente). Este instrumento fue traducido y adaptado en Chile por investigadores de la Universidad de La Frontera, Chile, quienes además estudiaron la confiabilidad y validez del instrumento. Los resultados indicaron un coeficiente de confiabilidad de 0.78 por medio del procedimiento test-retest. La validez concurrente se estimó aplicando el DEP ADO junto con la prueba AUDIT, obteniéndose una correlación de 0.71 usando la prueba $\mathrm{r}$ de Pearson, lo que indica que el instrumento posee adecuadas propiedades psicométricas (Molina, 2009; Vizcarra, 2007).

b) Cuestionario para evaluar la violencia de pareja en jóvenes. Construido por Vizcarra \& Poo (2007), el cual está compuesto por tres escalas: Violencia psicológica utiliza seis ítems, con una confiabilidad medida a través del coeficiente Alfa de Cronbach de 0,79. La Violencia física utiliza cinco ítems, con una confiabilidad Alfa de Cronbach de 0,69. La Violencia sexual está compuesta de tres ítems, con un Alfa de Cronbach de 0.64. Todas las escalas tienen el mismo formato, con cuatro opciones de respuesta, (nunca; 1 o 2 veces; 3 a 5 veces; 5 o más veces) con valores de 0 (nunca) a 3 ( 5 o más veces). En esta investigación se aplicó el coeficiente de confiabilidad Alfa de Cronbach al cuestionario total, resultando un valor de 0.80 . La escala de violencia psicológica, arroja un Alfa de 0.80; la escala de violencia física, 0.74, y la escala de violencia sexual, 0.32. Tanto la escala total como las subescalas mostraron adecuadas propiedades psicométricas, excepto la escala de violencia sexual, por lo que no fue utilizada en el análisis de resultados.

\section{Procedimiento}

En cada curso seleccionado, las investigadoras explicaron el objetivo del estudio a los jóvenes, solicitando su participación voluntaria. Se garantizó el anonimato y confidencialidad de la información. Posteriormente se leyó y entregó un consentimiento informado.

Con el fin de asegurar los resguardos éticos de la investigación, se les señaló a los participantes que, en caso de sentirse afectados por el contenido de las preguntas, se podían contactar con las investigadoras, quienes les realizarían una orientación preliminar y derivación correspondiente al Servicio Psicológico de la universidad.

Luego se aplicaron ambos cuestionarios, anexando preguntas relacionadas con variables socio-demográficas, como edad, género, carrera, etnia, nivel socioeconómico y participación en actividades extra académicas (deportivas, 
Tabla 1: Niveles de consumo de alcohol y otras drogas y género de los participantes

\begin{tabular}{ccc}
\hline Niveles de consumo & Género (\%) \\
\hline Leve & Hombre & Mujer \\
Moderado & 64 & 90 \\
Severo & 24 & 7 \\
Total & 12 & 3 \\
\hline
\end{tabular}

Nota: $\mathrm{n}=204, \chi^{2}=20,636, \mathrm{p}<0.001$

religiosas, artísticas, políticas u otras). La duración total de la aplicación de los instrumentos fue de cuarenta y cinco minutos aproximadamente.

Los resultados se analizaron a través del programa estadístico SPSS versión 15.0. Se aplicó la prueba de Kolmogorov-Smirnov y dado que la muestra no presentaba una distribución normal, se utilizaron pruebas no paramétricas.

\section{Resultados}

La muestra estuvo constituida por 81 hombres y 123 mujeres, y una persona que no consigna su género. El 96.1 $\%$ se encuentra entre los 18 a 24 años, y el $3.9 \%$ entre los 25 a 29 años. Un $26.8 \%$ pertenece a la carrera de Kinesiología, 24.4\% a Derecho, $17.1 \%$ a Psicología, $16.6 \%$ a Enfermería, $8.3 \%$ a Ingeniería Civil Informática y un $6.8 \%$ a Ingeniería Civil Industrial.

En cuanto al nivel socioeconómico, un $54.5 \%$ pertenece a un nivel medio, 39.5\% a nivel bajo y un $6 \%$ a nivel alto. En relación a la etnia a la que se autoadscriben, un $15.8 \%$ se declara mapuche, $83.7 \%$ no mapuche y $0.5 \%$ otras etnias. El nivel de participación en actividades extra-académicas en esta muestra fue de un $69.8 \%$. De éstos, $40 \%$ participa en actividades deportivas, un $28.3 \%$ en actividades religiosas, $18.5 \%$ en actividades artísticas, $6.3 \%$ en actividades políticas y un $6.8 \%$ en otras actividades.

En relación a la prevalencia del consumo de drogas, se obtuvo que el $81.3 \%$ ha consumido alcohol durante el último año, $48 \%$ ha consumido tabaco, $22.2 \%$ marihuana, $2.6 \%$ otros alucinógenos, $1.5 \%$ anfetaminas, $1 \%$ cocaína y $5.7 \%$ otras drogas (tranquilizantes, antidepresivos, entre otros).

Según los resultados del cuestionario DEP-ADO, el 80\% de la muestra presenta un consumo de alcohol y drogas a un nivel leve o no problemático ("verde"), un $13.2 \%$ a un nivel moderado ("amarillo"), y un $6.8 \%$ un nivel severo ("rojo"). Estos últimos niveles se consideran como un consumo abusivo y/o dependencia.

En referencia a la asociación entre consumo de drogas con variables socio-demográficas, se encontró una relación significativa con la edad ( $r h o=0.214 p=0.002)$, siendo el grupo de 18 a 24 años el que presenta mayor consumo ( $r h o=0.233, p=0.001)$. Respecto del género, los hombres presentan un mayor consumo en los niveles moderado y severo en comparación con las mujeres $\left(\chi^{2}=20.636\right.$, $p=0.001$ ) (ver Tabla 1).

Las otras variables analizadas, como carrera, etnia, nivel socioeconómico y participación en actividades sociales, no resultaron significativas.

En cuanto a la prevalencia de la violencia en el noviazgo, un $71,7 \%$ de los estudiantes encuestados declara haber experimentado al menos un episodio de violencia en su relación de pareja. Un $62 \%$ declara haber experimentado episodios de violencia psicológica y un $31.7 \%$ violencia física.

Al hacer la distinción entre víctima y agresor(a), se observa que el $41 \%$ refiere ser víctima de violencia psicológica, y un 30.2\% ejerce este tipo de violencia. En cuanto a la violencia física, un $16.1 \%$ señala ser víctima, y un $14.6 \%$ agresor(a).

También se analizó la correlación entre ser víctima de violencia y ser agresor(a), encontrando una asociación de $r h o=0.625(p=0.001)$.

Respecto a la violencia en el noviazgo y las variables sociodemográficas, se obtuvo una relación significativa con el género, siendo los hombres quienes más ejercen violencia en relación a las mujeres $\left(\chi^{2}=4.861, p=0.027\right)$. Sin embargo, al mismo tiempo, también son quienes más se reportan como víctimas, comparados con las mujeres. $\left(\chi^{2}=15.380, p=0.001\right)($ ver Tablas 2 y 3$)$

Las variables edad, carrera, etnia, nivel socioeconómico y participación en actividades sociales, no resultaron significativas.

En cuanto al objetivo general de la investigación, se obtuvo que existe una relación significativa entre el consumo de drogas y violencia en el noviazgo ( $r h o=0.164$, $p=0.019)$, confirmándose la hipótesis inicial. En relación al consumo de drogas y los tipos de violencia, se obtuvo una correlación significativa con la violencia psicológica ( $r h o=0.147, p=0.036)$ y con la violencia física $(r h o=0.158$, $p=0.023$ ) (ver Tabla 4). Al desagregar estos datos según víctima o agresor(a), se obtiene una relación significativa entre consumo de drogas y ser víctima de violencia psicológica $(r h o=0.191, p=0.006)$, y ser víctima de violencia física $(r h o=0.170, p=0.015)$ (ver Tabla 5$)$. 
Tabla 2: Agresión y género de los participantes

\begin{tabular}{ccc}
\hline Agresor & Género & \\
\hline & Hombre & Mujer \\
No & 59 & 105 \\
Sí & 22 & 18 \\
Total & 81 & 123 \\
\hline
\end{tabular}

Nota: $\mathrm{n}=204, \chi^{2}=4.861, \mathrm{p}<0.05$

Tabla 3: Victimización y género de los participantes

\begin{tabular}{ccc}
\hline Víctima violencia & Género \\
\hline & Hombre & Mujer \\
No & 47 & 102 \\
Sí & 34 & 21 \\
Total & 81 & 123 \\
\hline
\end{tabular}

Nota: $\mathrm{n}=204, \chi^{2}=15.380, \mathrm{p} \leq 0.001$

\section{Discusión}

Es relevante destacar la alta prevalencia del consumo de alcohol y drogas en los jóvenes universitarios chilenos, datos que son concordantes con los estudios de SENDA $(2004,2011)$ y el estudio de prevalencia de Vizcarra (2007). En esta investigación incluso, resultaron más altas las prevalencias de consumo de tabaco (48\%) y marihuana (22.2\%), y, a diferencia de la investigación de 2007, la categoría de consumo severo resultó más alta (6.8\%), lo cual resulta preocupante, en la medida que refleja dependencia y requiere intervención especializada.
Por otra parte, la prevalencia de la violencia en el noviazgo también resultó más alta que en estudios anteriores (Aguirre \& García, 1997; Vizcarra \& Poo, 2011). Sin embargo, estos resultados (62\% violencia psicológica y $31.7 \%$ violencia física) siguen la tendencia de estas investigaciones, que arrojan una mayor prevalencia de la violencia psicológica.

En relación al consumo de alcohol y otras drogas, y edad, los resultados concuerdan con las investigaciones de SENDA, que reportan que son los jóvenes de 19 a 25 años quienes presentan un mayor consumo, pudiendo transformarse éste en un consumo abusivo o dependencia.

Tabla 4: Consumo de alcohol y otras drogas y tipos de violencia

\begin{tabular}{lcc}
\hline & Consumo drogas $^{\text {a }}$ & $\mathrm{p}$ \\
\hline Violencia psicológica & 0.147 & $0.036^{*}$ \\
Violencia física & 0.158 & $0.023^{*}$ \\
Violencia total & 0.164 & $0.019^{*}$ \\
\hline
\end{tabular}

Nota: ${ }^{a}$ : Valores de Rho de Spearman ${ }^{*} \mathrm{p}<0.05$

Tabla 5: Consumo de alcohol y otras drogas y tipos de violencia, según victima/agresor(a)

\begin{tabular}{rcc}
\hline & Consumo drogas ${ }^{\text {a }}$ & $p$ \\
\hline Violencia psicológica víctima & 0.191 & $0.006^{* *}$ \\
Violencia psicológica agresor (a) & 0.129 & 0.065 \\
Violencia física víctima & 0.170 & $0.015^{*}$ \\
Violencia física agresor (a) & 0.091 & 0.193 \\
\hline
\end{tabular}

Nota: ${ }^{a}$ : Valores de Rho de Spearman ${ }^{*} \mathrm{p}<0.05{ }^{* *} \mathrm{p}<0.01$ 
Algunos autores han designado la etapa de 18 a 25 años como "adultez emergente", identificándola como un periodo de transición entre la adolescencia y la adultez, en el que se da un proceso de mayor exploración, con nuevos niveles de libertad y menos control parental, con una gran sensación de omnipotencia y pocos roles explícitos, lo que aumenta la probabilidad de involucrarse en conductas de riesgo, entre las cuales está el consumo de sustancias psicoactivas. Si a esto sumamos el contexto universitario, con altos grados de estrés, una cultura juvenil favorable al consumo y la falta de motivación académica en algunos estudiantes, se tiene que ambos factores, edad y vida universitaria, incrementan el riesgo de consumo de sustancias lícitas e ilícitas, lo cual atenta contra el proyecto de vida del joven y su bienestar futuro (Martínez, Zuzulich, Contreras, Cantillano, Gyslin \& Pedrals, 2010).

En cuanto al género y el consumo de alcohol y otras drogas, el mayor consumo en varones es concordante con los estudios sobre consumo en jóvenes (SENDA, 2009, 2011), y específicamente en jóvenes universitarios (SENDA, 2004, 2006; Vizcarra, 2007). Esto puede deberse a la existencia de patrones culturales que favorecen una mayor permisividad en el consumo para los varones (Guzmán et al., 2009). Sin embargo, como señalan Romero, Santander, Hitschelfd, Labbé \& Zamora (2009), se ha observado en los últimos estudios una tendencia a igualar el consumo entre hombres y mujeres, por lo que habría que contemplar con cautela esta relación.

En cuanto a la violencia en el noviazgo y las variables sociodemográficas, el género resultó ser una variable significativa. Al igual que en la violencia de pareja en adultos, los hombres son quienes ejercen violencia en mayor proporción. No obstante, también resultó significativa la cantidad de hombres que se reportan como víctimas, incluso en una proporción mayor que las mujeres. Este hallazgo estaría relacionado con la característica de violencia cruzada que se da en la violencia en el noviazgo, donde las mujeres con mayor frecuencia ejercen violencia, predominantemente psicológica y física leve, en contra de sus parejas, a diferencia de la violencia de pareja adulta en que la violencia tiende a ser más bien unilateral, hacia la mujer (Aguirre y García, 1997; Poo \& Vizcarra, 2008; INJUV, 2009). La alta correlación víctima-victimario(a) encontrada, confirma esta característica de bidireccionalidad en la violencia en parejas jóvenes. Se ha señalado que podría influir una mayor equidad de poder en las parejas jóvenes, como ciertos patrones culturales, donde existe menor sanción social para la agresión femenina (Servicio Nacional de la Mujer, Gobierno de Chile, 2004; Vizcarra \& Poo, 2008).

En cuanto a la relación entre consumo de drogas y violencia en el noviazgo, se confirma la existencia de una relación significativa y directa, aunque la intensidad de esta relación es baja. Como se mencionó en los antecedentes teóricos, en ambas variables subyacen factores de riesgo comunes, como ser testigo o víctima de violencia intrafamiliar en la infancia, disfuncionalidad familiar, contexto comunitario de violencia y creencias estereotipadas respecto de los roles de género (Hernando, 2007: Vézina \& Hebert, 2007). Otros autores mencionan a estos fenómenos como parte de un "síndrome de comportamiento problema" en el que ambos covarían junto a otros comportamientos de riesgo (Jessor, 1993; Muñoz -Rivas et al., 2010), por lo que se comprende que la correlación no sea alta, ya que forman parte de una constelación de comportamientos de riesgo.

La relación entre consumo de drogas y violencia física y psicológica resulta concordante con investigaciones similares realizadas en otros países (González, 2008; Guzmán et al., 2009; Muñoz-Rivas et al., 2010). Es decir, a mayor consumo (moderado a severo), se observará una mayor violencia, asociación se fundamentaría en el hecho de que el consumo de alcohol y otras sustancias altera la percepción de los patrones de interacción y comunicacionales en la pareja, por lo que es más probable que surjan comportamientos violentos. Por otra parte el consumo de sustancias altera el juicio de realidad, impidiendo reconocer situaciones de peligro y disminuyendo las conductas de autoprotección, por lo que facilitaría la probabilidad de ser víctima de violencia, especialmente física y sexual, lo cual fue corroborado en este estudio (Foshee et al., 2007; González, 2008; Guzmán et al., 2009; Vézina \& Hébert, 2007).

En el presente estudio no se observaron relaciones significativas entre consumo y perpetración de violencia, lo que podría estar relacionado con el tamaño muestral y la menor cantidad de participantes que reconocieron haber ejercido violencia. Si bien es posible que exista menor perpetración de violencia que victimización en estudiantes universitarios, no se puede desconocer la posible influencia de la deseabilidad social en la falta de reconocimiento. Con el propósito de controlar este aspecto se construyó una escala, la cual, en su aplicación piloto mostró propiedades psicométricas insuficientes, por lo que se descartó su aplicación a la muestra definitiva. Este aspecto debería considerarse en futuras investigaciones (González, 2008).

Una posible explicación a la ausencia de asociación entre consumo y ejercicio de la violencia es la poca variabilidad de la muestra, ya que la mayoría de los sujetos respondió negativamente a la violencia sexual y consumo severo de drogas, lo cual es esperable en una muestra no clínica. Esto probablemente afectó la medición de la violencia sexual, por lo cual no se pudo analizar su prevalencia ni su relación con el consumo de alcohol y drogas. A partir de lo anterior, se sugiere en futuras investigaciones ampliar la muestra, incluyendo población clínica (jóvenes en centros de rehabilitación, $\mathrm{y} / \mathrm{o}$ consultantes en salud mental por violencia en el noviazgo) y mediciones de la violencia sexual para obtener una descripción más completa de estos fenómenos y mayor representatividad de los resultados. Otra sugerencia de investigación, es la utilización de metodologías cualitativas, 
con el fin de conocer las percepciones, creencias y visión de los jóvenes acerca de estas temáticas y su relación. Esto con el fin de lograr una comprensión más profunda y por ende intervenciones más pertinentes.

En cuanto a los alcances de esta investigación, al ser una de las primeras que indaga la relación entre violencia en el noviazgo y el consumo de drogas en Chile, abre un camino para continuar profundizando en estos temas, tanto desde una perspectiva cuantitativa como cualitativa.

Como sugerencias para la intervención, por tanto, se puede señalar que estos resultados son relevantes para confirmar la coincidencia de ambos fenómenos como situaciones importantes que ocurren en los jóvenes, y que deben ser abordadas integralmente junto con otras conductas de riesgo, ya que actualmente se estarían abordando de manera parcializada, disminuyendo la efectividad de las intervenciones, tanto preventivas como remediales (Muñoz-Rivas et al., 2010).

Es necesario intervenir de manera preventiva en los primeros años de universidad, favoreciendo la reflexión y problematización en torno al consumo de drogas lícitas e ilícitas, y sus consecuencias. Asimismo es necesario problematizar con respecto a la violencia en el noviazgo y las creencias culturales asociadas a este tipo de violencia, indicando sus consecuencias a largo plazo y las características específicas de este tipo de violencia de pareja. Esto es particularmente relevante en jóvenes universitarios, ya que es en estas instituciones de educación superior donde se debe promover el desarrollo personal, académico y social de sus estudiantes, para lograr una futura sociedad más saludable, auténtica y efectiva (Martínez et al., 2010).

\section{Referencias}

Aguirre, A. M., \& García, M. (1997).Violencia prematrimonial: Un estudio exploratorio en universitarios. Ultima Década, 6, 229-248.

Espada, J., Méndez, J., Griffin, K., \& Botvin, G. (2003). Adolescencia: Consumo de alcohol y drogas. Papeles de Psicólogos, 84, 9-17.

Foshee, V.A., Bauman, K.E., Linder, G.F., Rice, J., \& Wilcher, R. (2007). Typologies of adolescent dating violence: Identifying typologies of adolescent dating violence perpetration. Journal of Interpersonal Violence, 22, 498.

González, M.P., Muñoz, M., \& Graña, J.L. (2003).Violencia en las relaciones de pareja en adolescentes: Una revisión. Psicopatología Clínica Legal y Forense, 3, 23-39.

González, M.P. (2008). Violencia en las relaciones de noviazgo entre jóvenes adolescentes de la comunidad de Madrid. (Tesis inédita de Doctorado). Facultad de Psicología, Universidad Complutense de Madrid, España.

Guzmán, F., Esparza, S., Alcántara, S., Escobedo, I., \& Henggeler, T. (2009). Consumo de alcohol en jóvenes y su relación con la violencia psicológica en el noviazgo. Revista electrónica Saúde Mental, Alcool e Droga. 5, (2), 3. Recuperado de: http://www.eerp.usp.br/resmad/ artigos.asp.

Hernando, A. (2007). La prevención de la violencia de género en adolescentes. Una experiencia en el ámbito educativo. Apuntes de Psicología, $25,325-340$

Instituto Nacional de la Juventud (INJUV) (2009). Sexta Encuesta Nacional de la Juventud. Santiago, Chile. Recuperado de: http://www. injuv.gob.cl/modules.php.
Jessor, R. (1993). Successful adolescent development among youth in high risk settings. American Psychologist, 148, 117-126.

Landry, M., Tremblay, J., Guyon, L., Bergeron, J., \& Brunelle, N. (2004). La Grille de dépistage de la consommation problématique d'alcool et de drogues chez las adolescents et les adolescentes (DEP-ADO): Devéloppement et qualités psychométriques. Drogues, santé et société, 3,19-37.

Martínez, D., Zuzulich, M., Contreras, L., Cantillano, V., Gysling, K., \& Pedrals, N. (2010). Hasta cuándo hablamos de las drogas: Estrategias preventivas en contextos universitarios. Santiago: Ediciones Universidad Católica de Chile.

Molina, J.L. (2009). Caracterización psicométrica de las escalas DEP - ADO y AUDIT para detectar consumo de drogas en adolescentes. (Tesis inédita de Maestría en Psicología). Universidad de La Frontera, Temuco, Chile.

Muñoz-Rivas, M., Gámez-Guadix, M., Graña, J.L., \& Fernández, L. (2010).Violencia en el noviazgo y consumo de alcohol y drogas ilegales entre adolescentes y jóvenes españoles. Adicciones, 22, 125-134.

Poo, A.M., \& Vizcarra, B. (2008).Violencia de pareja en jóvenes universitarios.Terapia Psicológica, 26, 81- 88.

Rees, R., \& Valenzuela, A. (2003). Características individuales y de la estructura familiar de un grupo de adolescentes abusadores de alcohol y/o marihuana. Revista Chilena de Neuropsiquiatría, 41, 173-185.

Rivera- Rivera, L., Allen, B., Rodríguez-Ortega, G., Chávez-Ayala, R., \& Lazcano-Ponce, E., (2006).Violencia durante el noviazgo, depresión y conductas de riesgo en estudiantes femeninas. Revista de Salud Pública de México, 48, 288-296.

Rodríguez, J., Fernández, A. M., Hernández, E., \& Ramírez, S. (2006). Conductas agresivas, consumo de drogas e intentos de suicidio en jóvenes universitarios. Terapia Psicológica, 24, 63-69.

Romero, M. I., Santander, J., Hitschfeld, M., Labbé, M., \& Zamora, V. (2009). Consumo de tabaco y alcohol entre los estudiantes de medicina de la Pontificia Universidad Católica de Chile. Revista Médica de Chile, 137, 361-368.

Servicio Nacional para la Prevención y Rehabilitación del Consumo de Drogas y Alcohol (SENDA) (2011). Noveno Estudio Nacional de Drogas en Población General de Chile. Santiago, Chile. Recuperado de: http://www.conacedrogas.gob.cl/wpcontent/uploads/2011/03/2010 InformeEjecutivo_Noveno_Estudio_General.pdf.

Servicio Nacional para la Prevención y Rehabilitación del Consumo de Drogas y Alcohol (SENDA) (2009). Octavo Estudio Nacional de Drogas en Población General de Chile. Recuperado de: www.conacedrogas. $\mathrm{cl} / . . . /$ resumen/informe_VIII_estudio_drogas_poblaciongeneral_junio2009 pdf.

Servicio Nacional para la Prevención y Rehabilitación del Consumo de Drogas y Alcohol (SENDA) (2006). Séptimo Estudio Nacional de Drogas en Población General de Chile, Recuperado de: http://www. conacedrogas.cl/inicio/pdf/

Servicio Nacional para la Prevención y Rehabilitación del Consumo de Drogas y Alcohol, (SENDA) (2004). Informe sobre uso de drogas en estudiantes de la educación superior. Recuperado de: http://www. conacedrogas.cl/Uso_drogas_estudiantes_educacion_superior_CONACE2004.pdf.

Servicio Nacional de la Mujer (SERNAM) (2004). Análisis de la violencia en las relaciones de pareja entre jóvenes, Documento de trabajo $\mathrm{N}^{\circ} 105$, Chile. Recuperado de: http://www.sernam.cl/cedocvi/estudios/pdf.

Vézina, J., \& Hébert, M. (2007). Risk factors for victimization in romantic relationships of young women. Trauma, Violence \& Abuse, 8, 33-66.

Vizcarra, M.B. (2007). Patrones de consumo de alcohol y drogas en alumnos de primer año de la Universidad de la Frontera. Informe Final Proyecto Educación Superior. Universidad de la Frontera - CONACE. Temuco. Chile.

Vizcarra M. B., \& Poo, A. M. (2011). Violencia de pareja en estudiantes universitarios del sur de Chile. Universitas Psychologica, 10, 89-98.

Wolfe, D., \& Feiring, C. (2000). Dating violence through the lens of adolescent romantic relationships. Child maltreatment, 5, 360. 